\title{
Tanshinone IIA inhibits human hepatocellular carcinoma J5 cell growth by increasing Bax and caspase 3 and decreasing CD31 expression in vivo
}

\author{
SU-YU CHIEN $^{1}$, SHOU-JEN KUO ${ }^{2,3}$, YAO-LI CHEN ${ }^{2,3}$, DAR-REN CHEN $^{2,3}$, \\ CHUN-YUAN CHENG ${ }^{2,3}$ and CHIN-CHENG SU ${ }^{2,3,4}$
}

\author{
Departments of ${ }^{1}$ Pharmacy and ${ }^{2}$ Surgery, Changhua Christian Hospital, Changhua City, Changhua; \\ ${ }^{3}$ Institute of Medicine, Chung Shan Medical University, Taichung; \\ ${ }^{4}$ Mingdao University, Pee Tow, Changhua, Taiwan, R.O.C.
}

Received June 9, 2011; Accepted October 10, 2011

DOI: $10.3892 / \mathrm{mmr} .2011 .631$

\begin{abstract}
Tanshinone IIA (Tan-IIA) decreases the viability of human hepatocellular carcinoma (HCC) cells through the induction of apoptosis in vitro. However, there are no reports that Tan-IIA is capable of inhibiting J5 HCC cell growth in vivo. In this study, J5 cells were implanted directly into nude SCID mice which were divided randomly into four groups to be treated with vehicle, Tan-IIA ( $30 \mathrm{mg} / \mathrm{kg}$ of body weight, Q.week days 3 and 5), 5-FU (30 mg/kg of body weight, Q.week day 1) or Tan-IIA (30 mg/kg of body weight, Q.week days 3 and 5) plus 5 -FU (30 mg/kg of body weight, Q.week day 1). Each agent was injected intraperitoneally, with treatment starting 4 weeks after inoculation with J5 cells. Treatment with Tan-IIA $30 \mathrm{mg} / \mathrm{kg}$ or with $30 \mathrm{mg} / \mathrm{kg}$ of $5-\mathrm{FU}$ resulted in a reduction in tumor size and weight compared with the control group. The protein expression of Bax and caspase- 3 in the J5 xenograft tumors treated with Tan-IIA $30 \mathrm{mg} / \mathrm{kg}$ or with $30 \mathrm{mg} / \mathrm{kg}$ of 5 -FU was upregulated, whereas that of CD31 was downregulated compared with the control group. These findings indicate that Tan-IIA may inhibit tumor growth in a J5 xenograft animal model by increasing Bax and caspase 3 and decreasing CD31 expression in vivo.
\end{abstract}

\section{Introduction}

Hepatocellular carcinoma is the leading cause of cancer-related death in men (41.4/100,000 deaths) in Taiwan (1). HCC is chemoresistant to a number of available chemotherapeutic agents (2). Numerous prescription drugs in use for cancer treatment are derived from plants (3). Tanshinone IIA (Tan-IIA) is

Correspondence to: Dr Chin-Cheng Su, Department of Surgery, Comprehensive Breast Cancer Center, Laboratory of Integrative Medicine Cancer Research, Changhua Christian Hospital, 135 Nan-Hsiao Street, Changhua City, Changhua 500-06, Taiwan, R.O.C. E-mail: succ.maeva@msa.hinet.net

Key words: tanshinone IIA, J5 cells, Bax, caspase 3, CD31, in vivo an extract from Salviae Miltiorrhizae radix $(4,5)$, and its antiinflammatory activities $(6,7)$, antitumor activity in numerous human cancer cell types (8-10) and anti-oxidant properties $(11,12)$ have been well documented. In addition, it has been extensively reported that Tan-II A inhibits cell proliferation and induces apoptosis in hepatocellular carcinoma cell lines (13-15). Tan-IIA effectively inhibited the invasion and metastasis of HCC cells in vitro and in vivo (16). Our previous study revealed that Tan-IIA inhibited hep-J5 cell growth in vitro via induction of apoptosis and increase in protein expression of calreticulin, caspase 12 and GADD153 (17). However, the anticancer effects of Tan-IIA on hep-J5 cells in vivo are not yet fully understood. The present study focused on the anticancer effect of Tan-IIA in vivo in SCID mice, using a human hepatocellular carcinoma xenograft model of J5 cells. Additionally, since 5-fluorouracil (5-FU) is one of the chemotherapeutic agents for HCC, we also examined whether there were any synergistic effects between Tan-IIA and 5-FU in this study.

\section{Materials and methods}

Chemicals. Tan-IIA (molecular formula: $\mathrm{C}_{19} \mathrm{H}_{18} \mathrm{O}_{3}$, purity $>96 \%$ by HPLC) was purchased from Herbasin Co. (Shenyang, China). Corn oil, aprotinin, antipain, sodium deoxycholate, leupeptin, propidium iodide (PI), sodium orthovanadate, Triton $\mathrm{X}-100$, Tris- $\mathrm{HCl}$, ribonuclease-A and trypan blue were obtained from Sigma Chemical Co. (St. Louis, MO, USA).

Cell culture. The human hepatocellular carcinoma J5 cell line was kindly provided by Dr H.J. Harn (Graduate Institute of Cancer Biology and Center for Molecular Medicine, China Medical University and Hospital, Taichung, Taiwan).

The J5 cells were maintained in RPMI-1640 medium containing $10 \% \mathrm{FBS}, 1 \%$ penicillin-streptomycin $(10,000 \mathrm{U} / \mathrm{ml}$ penicillin; $10 \mathrm{mg} / \mathrm{ml}$ streptomycin) in a $37^{\circ} \mathrm{C}$ humidified atmosphere containing $5 \% \mathrm{CO}_{2}$.

In vivo J5 cell tumor xenograft model. A total of 24 3-week-old male nude SCID mice (NOD.CB 17-Prkdcscid/Tcu) were 


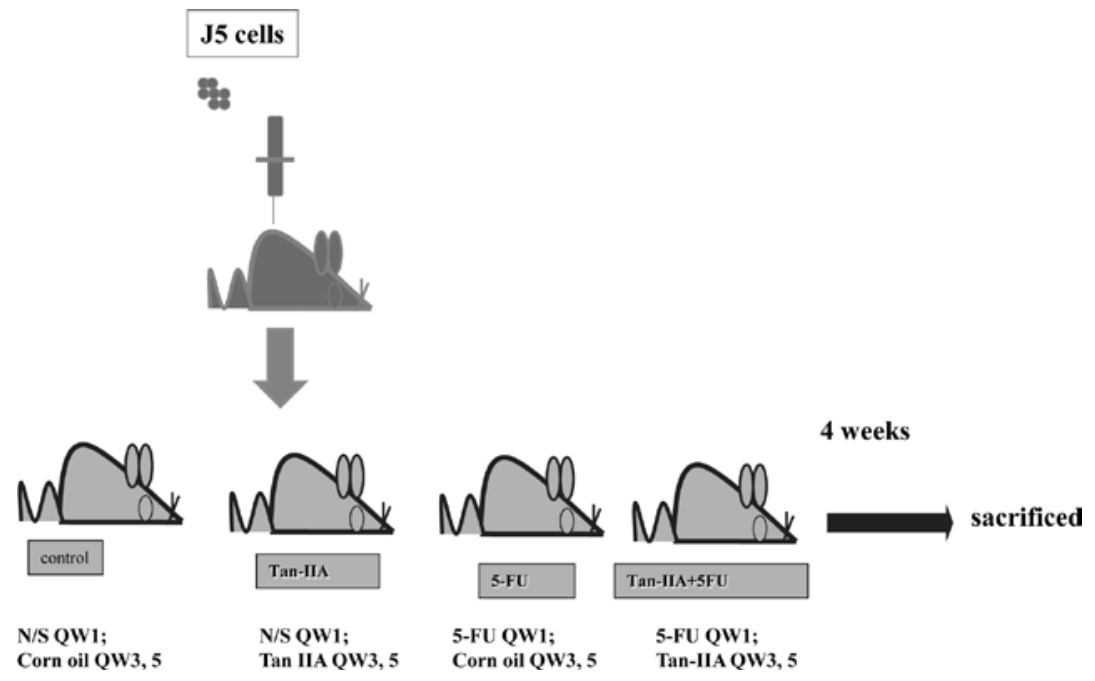

Figure 1. Experimental design of the xenograft animal model. J5 cell tumor xenograft in NOD.CB 17-Prkdcscid/Tcu mice. J5 cells were implanted directly into nude SCID mice, which were divided randomly into four groups and treated with vehicle, Tan-IIA (30 mg/kg of body weight, Q.week days 3 and 5), 5-FU (30 mg/kg of body weight, Q.week day 1) or Tan-IIA (30 mg/kg of body weight, Q.week days 3 and 5) plus 5-FU (30 mg/kg of body weight, Q.week day 1). Each agent was injected intraperitoneally, with treatment starting 4 weeks after inoculation with the J5 cells, and the hep-J5 cell xenograft tumor volumes were measured. Mice were sacrificed by $\mathrm{CO}_{2}$ inhalation 35 days later, after which hep-J5 cell xenograft tumors were dissected and individually weighed.
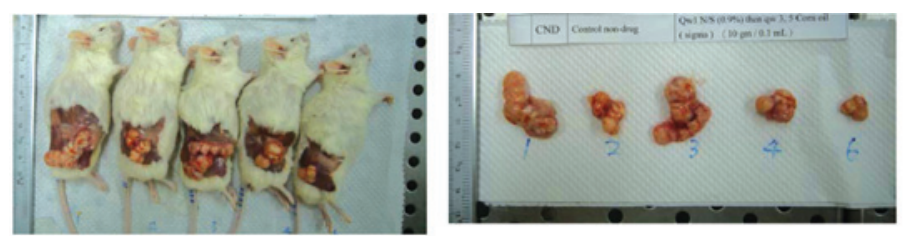

\section{Control}
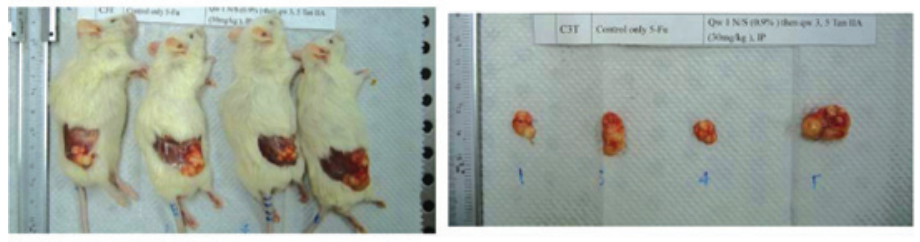

C3T
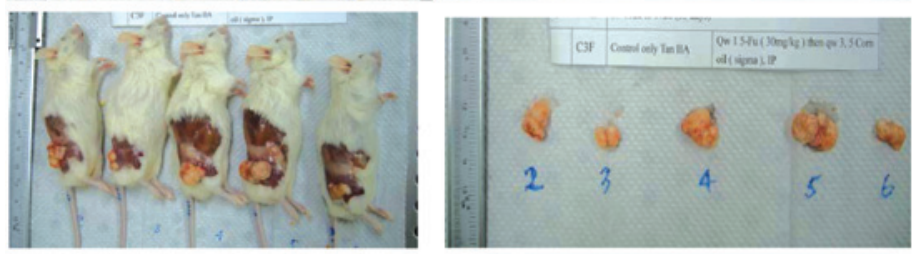

$\mathrm{C} 3 \mathrm{~F}$
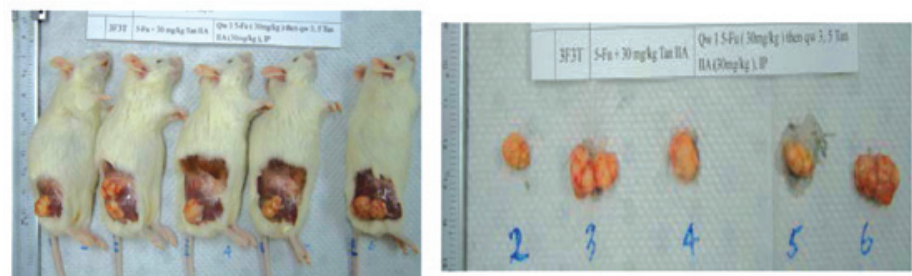

$3 \mathrm{~F} 3 \mathrm{~T}$

Figure 2. SCID mice bearing J5 cell xenograft tumors were treated with vehicle (control group), Tan-IIA [30 mg/kg of body weight, Q.week day 3, 5 (C3T group)], 5 -FU [30 mg/kg of body weight, Q.week day 1 (C3F group)] or Tan-IIA (30 mg/kg of body weight, Q.week days 3 and 5) plus 5-FU (30 mg/kg of body weight, Q.week day 1) (3F3T group) for 4 weeks and then sacrificed with $\mathrm{CO}_{2}$ inhalation. Xenograft tumors were subsequently dissected.

xenografted with human hepatocellular carcinoma (HCC) $\mathrm{J} 5$ cells $\left(3 \times 10^{6} / 0.2 \mathrm{ml}\right)$ and maintained in a pathogen-free environment (Laboratory Animal Center of Tzu Chi University, Hualien, Taiwan). On day 28, the mice were divided randomly into four groups (6 mice/group) to be treated with Tan-IIA $(30 \mathrm{mg} / \mathrm{kg}$ of body weight, dissolved in corn oil, Q.week days 3 and 5), 5-FU (30 mg/kg of body weight, dissolved in normal saline, Q.week day 1) or Tan-IIA (30 mg/kg of body weight, Q.week days 3 and 5) plus $5-\mathrm{FU}$ (30 mg/kg of body weight, Q.week day 1). As a control, xenografted tumors were separately treated with normal saline $(0.1 \mathrm{ml} / 10 \mathrm{~g}$ body weight, Q.week day 1$)$ and corn oil $(0.1 \mathrm{ml} / 10 \mathrm{~g}$ body weight, Q.week days 3 and 5). Each agent was injected intraperitoneally. At the end of the 4-week dosing schedule, the SCID mice 

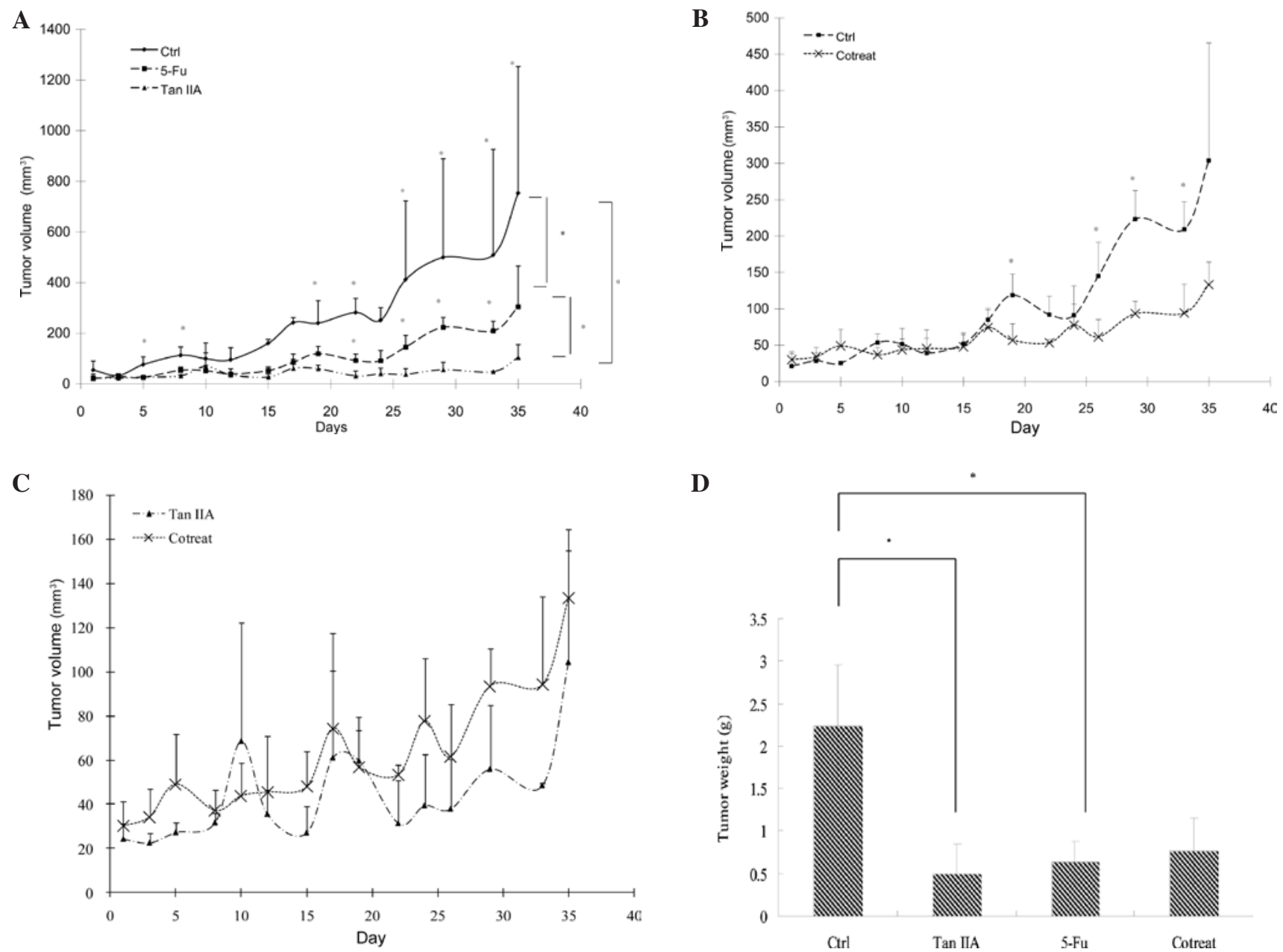

Figure 3. J5 cells were implanted directly into nude SCID mice which were divided randomly into four groups to be treated with vehicle (control group), Tan-IIA [30 mg/kg of body weight, Q.week days 3 and 5 (C3T group)], $5-\mathrm{FU}$ [30 mg/kg of body weight, Q.week day 1 (C3F group)] or Tan-IIA (30 mg/kg of body weight, Q.week days 3 and 5) plus 5-FU (30 mg/kg of body weight, Q.week day 1) (3F3T group). Each agent was injected intraperitoneally. After xenograft transplantation, mice exhibiting tumors were monitored, and the tumor size was measured once every 2 days using calipers. The tumor volume in each animal was estimated according to the formula: Tumor volume $\left(\mathrm{mm}^{3}\right)=\mathrm{Lx} \mathrm{W} / 2$ (where $\mathrm{L}$ is the length and W is the width) with the final measurement taken 5 weeks after tumor cell inoculation. Mice were treated for 28 days, then sacrificed by $\mathrm{CO}_{2}$ inhalation. The hep-J5 cell xenograft tumors were dissected and individually weighed. (A-C) Treatment with Tan-IIA $30 \mathrm{mg} / \mathrm{kg}$ or with $30 \mathrm{mg} / \mathrm{kg}$ of 5 -FU resulted in a reduction in tumor size and (D) weight compared with the control group. ${ }^{*} \mathrm{P}<0.05$

were sacrificed by $\mathrm{CO}_{2}$ inhalation on day 35 . Following xenograft transplantation, mice exhibiting tumors were monitored, and tumor size was measured once every 2 days using calipers. The tumor volume for each animal was estimated according to the following formula: Tumor volume $\left(\mathrm{mm}^{3}\right)=\mathrm{L} \mathrm{x} \mathrm{W} / 2$ (where $\mathrm{L}$ is the tumor length and $\mathrm{W}$ is the width) with the final measurement taken 5 weeks after tumor cell inoculation. At the same time, the body weight of each animal was measured once every 2 days. The xenograft tumors were dissected and weighed individually before the proteins were extracted for Western blotting. The protein expression of Bax, caspase- 3 and CD31 was measured by Western blotting. Immunoreactive bands were scanned and analyzed using a digital scanning densitometer (Molecular Dynamics, Sunnyvale, CA, USA). The experimental design is shown in Fig. 1.

Statistical analysis. Each value represents the mean \pm SD. The control and experimental animal groups were compared using the Student's t-test. $\mathrm{P}<0.05$ was considered to denote statistical significance.

\section{Results and discussion}

The results indicated that Tan-IIA and 5-FU significantly reduced hep-J5 cell xenograft tumor size. Representative animals treated with Tan-IIA relative to the control are shown in Fig. 2. Tan-IIA treatment significantly decreased tumor volume (Fig. 3A-C) and weight (Fig. 3D) compared with the control. The percent inhibition of tumor weight is shown in Table I. None of the treatments altered the body weight significantly (data not shown). Tumors were only observed at the inoculation sites. Based on these in vivo experiments, it can be seen that Tan-IIA at $30 \mathrm{mg} / \mathrm{kg}$ can inhibit tumor growth in a J5 xenograft model. In the present study, tumors in mice that received Tan-IIA alone were approximately 55\% smaller than those of the control group (Fig. 3A). In the 5-FU treatment and Tan-IIA plus 5-FU groups, tumors continued to grow slowly compared with the control group, indicating that complete regression of J5 cell xenografts was not achieved using a single treatment agent, and therefore that multiple treatments may be required to achieve a complete response. 
A
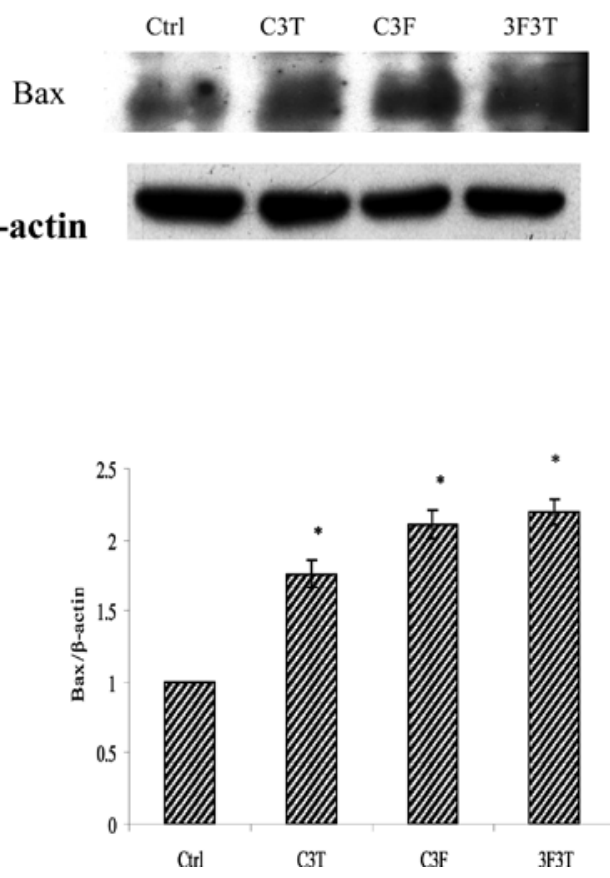

C
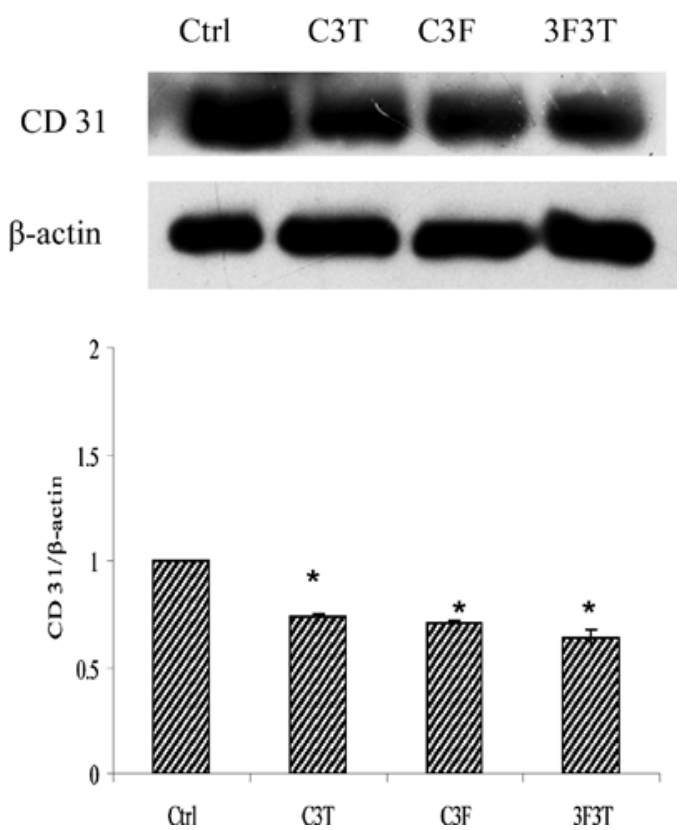

Figure 4. Protein expression of Bax, caspase 3 and CD31 in hep-J5 cell xenograft tumors. The xenograft tumors were dissected and weighed individually, after which the proteins, Bax, caspase-3 and CD31, were extracted using Western blotting. The protein expression of (A) Bax and (B) caspase 3 in xenograft tumors treated with Tan-IIA and/or 5-FU was upregulated when compared to the control group. (C) The protein expression of CD31 in xenograft tumors treated with Tan-IIA (C3T group), 5-FU (C3F group) or their combination (3F3T group) was downregulated when compared to the control group. ${ }^{*} \mathrm{P}<0.05$ significant difference compared to the controls.

However, our results revealed that Tan-IIA possesses therapeutic potential in HCC J5 cells in vivo. This is in agreement with other studies (18). However, the combination of Tan-IIA and 5-FU was not capable of producing any synergistic effects in $\mathrm{J} 5$ cells in vivo (Fig. $3 \mathrm{C}$ and D). Our results also revealed that the protein expression of Bax (Fig. 4A) and caspase
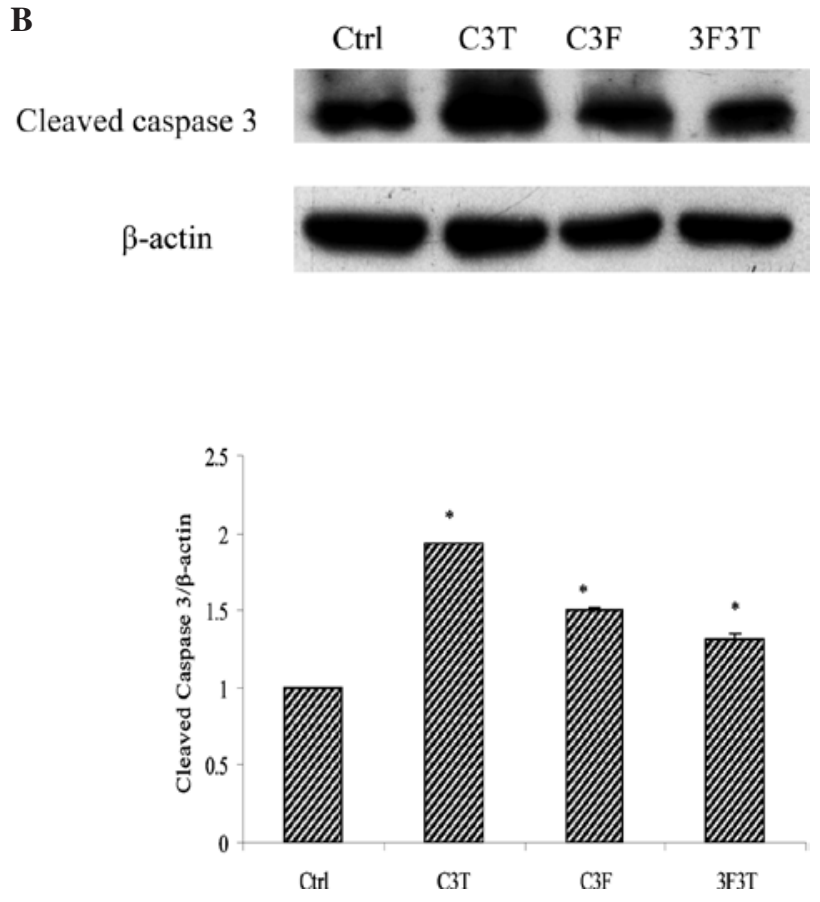

Table I. Inhibitory effect of Tan-IIA on growth of hep-J5 tumor xenografts in SCID mice.

\begin{tabular}{lcc}
\hline Treatment & Tumor weight $(\mathrm{g})$ & Inhibition $(\%)$ \\
\hline Control & $2.23 \pm 0.72$ & \\
Tan-IIA & $0.50 \pm 0.35$ & $77.61^{\mathrm{a}}$ \\
5-FU & $0.63 \pm 0.25$ & $71.64^{\mathrm{a}}$ \\
Co-treated & $0.77 \pm 0.39$ & 65.67
\end{tabular}

Control and Tan-II, 5-FU, cotreatment groups were compared and analyzed using the Student's t-test. ${ }^{\mathrm{a}} \mathrm{p}<0.05$.

3 (Fig. 4B) in xenograft tumors treated with Tan-IIA and/or 5-FU was upregulated when compared to the control group. This is in agreement with our previous in vitro study and others $(13,17)$. The protein expression of CD31 (Fig. 4C) in xenograft tumors treated with Tan-IIA and/or 5-FU was downregulated when compared to the control group. This result indicated that Tan-IIA and/or 5-FU did not increase the vascularity in hep-J5 xenograft tumors. The present study provides the first report of the efficacy of Tan-IIA against tumors in an in vivo xenograft of human HCC J5 cells in SCID mice.

\section{Acknowledgements}

This study was supported by grant CCMP97-RD-041 from the Committee on Chinese Medicine and Pharmacy, Department of Health, Executive Yuan, Taiwan.

\section{References}

1. Department of Health, Executive Yuan: Statistics of Causes of Death, 2007. Taipei, Taiwan R.O.C, pp34-46, 2008.

2. Leung TW and Johnson PJ: Systemic therapy for hepatocellular carcinoma. Semin Oncol 28: 514-520, 2001. 
3. Kucuk O: Chemoprevention of prostate cancer. Cancer Metastasis Rev 21: 111-124, 2002.

4. Che AJ, Zhang JY, Li CH, Chen XF, Hu ZD and Chen XG: Separation and determination of active components in Radix Salviae miltiorrhizae and its medicinal preparations by nonaqueous capillary electrophoresis. J Sep Sci 27: 569-575, 2004.

5. Zhou L, Zuo Z and Chow MS: Danshen: An overview of its chemistry, pharmacology, pharmacokinetics, and clinical use. J Clin Pharmacol 45: 1345-1359, 2005.

6. Jang SI, Kim HJ, Kim YJ, Jeong SI and You YO: Tanshinone IIA inhibits LPS-induced NF-kappaB activation in RAW 264.7 cells: possible involvement of the NIK-IKK, ERK1/2, p38 and JNK pathways. Eur J Pharmacol 542: 1-7, 2006.

7. Li W, Li J, Ashok M, Wu R, Chen D, Yang L, Yang H, Tracey KJ, Wang P, Sama AE and Wang H: A cardiovascular drug rescues mice from lethal sepsis by selectively attenuating a late-acting proinflammatory mediator, high mobility group box 1. J Immunol 78: 3856-3864, 2007.

8. Liu JJ, Lin DJ, Liu PQ, Huang M, Li XD and Huang RW: Induction of apoptosis and inhibition of cell adhesive and invasive effects by tanshinone IIA in acute promyelocytic leukemia cells in vitro. J Biomed Sci 13: 813-823, 2006.

9. Su CC and Lin YH: Tanshinone IIA inhibits human breast cancer cells through increased Bax to Bcl-xL ratios. Int $\mathrm{J}$ Mol Med 22: 357-361, 2008.

10. Su CC, Chen GW, Kang JC and Chan MH: Growth inhibition and apoptosis induction by tanshinone IIA in human colon adenocarcinoma cells. Planta Med 74: 1357-1362, 2008.
11. Lin R, Wang WR, Liu JT, Yang GD and Han CJ: Protective effect of tanshinone IIA on human umbilical vein endothelial cell injured by hydrogen peroxide and its mechanism. J Ethnopharmacol 108: 217-222, 2006.

12. Wang AM, Sha SH, Lesniak W and Schacht J: Tanshinone (Salviae miltiorrhizae extract) preparations attenuate aminoglycoside-induced free radical formation in vitro and ototoxicity in vivo. Antimicrob Agents Chemother 47: 1836-1841, 2003.

13. Yuan SL, Wei YQ, Wang XJ, Xiao F, Li SF and Zhang J: Growth inhibition and apoptosis induction of tanshinone II-A on human hepatocellular carcinoma cells. World J Gastroenterol 10: 2024-2028, 2004.

14. Zhai XM, He SX, Ren MD, Chen JH, Wang ZL, Han M and Hou HL: Effect of Tanshinone II A on expression of EGF and EGFR in hepatocellular carcinoma cell line SMMC-7721. Zhejiang Da Xue Xue Bao Yi Xue Ban 38: 163-169, 2009.

15. Lee WY, Liu KW and Yeung JH: Reactive oxygen species-mediated kinase activation by dihydrotanshinone in tanshinones-induced apoptosis in HepG2 cells. Cancer Lett 285: 46-57, 2009.

16. Yuxian X, Feng T, Ren L and Zhengcai L: Tanshinone II-A inhibits invasion and metastasis of human hepatocellular carcinoma cells in vitro and in vivo. Tumori 95: 789-795, 2009.

17. Cheng CY and Su CC: Tanshinone IIA inhibits Hep-J5 cells by increasing calreticulin, caspase 12 and GADD153 protein expression. Int J Mol Med 26: 379-385, 2010.

18. Li Q, Wang Y, Feng N, Fan Z, Sun J and Nan Y: Novel polymeric nanoparticles containing tanshinone IIA for the treatment of hepatoma. J Drug Target 16: 725-732, 2008. 